\title{
An ALMA view of the interstellar medium of the $z=4.77$ lensed starburst SPT-S J213242-5802.9
}

\author{
M. Béthermin ${ }^{1}$, C. De Breuck ${ }^{1}$, B. Gullberg ${ }^{1}$, M. Aravena ${ }^{2}$, M. S. Bothwell ${ }^{3}$, S. C. Chapman ${ }^{4}$, A. H. Gonzalez ${ }^{5}$, \\ T. R. Greve ${ }^{6}$, K. Litke $^{8}$, J. Ma ${ }^{5}$, M. Malkan ${ }^{7}$, D. P. Marrone ${ }^{8}$, E. J. Murphy ${ }^{9}$, J. S. Spilker ${ }^{8}$, A. A. Stark ${ }^{10}$, \\ M. Strandet ${ }^{11}$, J. D. Vieira ${ }^{12}$, A. Weiß ${ }^{11}$, and N. Welikala ${ }^{13}$ \\ ${ }^{1}$ European Southern Observatory, Karl Schwarzschild Straße 2, 85748 Garching, Germany \\ e-mail: mbetherm@eso.org \\ 2 Núcleo de Astronomía, Facultad de Ingeniería, Universidad Diego Portales, Av. Ejército 441, Santiago, Chile \\ 3 Cavendish Laboratory, University of Cambridge, JJ Thompson Ave, Cambridge CB3 OHA, UK \\ ${ }^{4}$ Dalhousie University, Halifax, Nova Scotia, Canada \\ 5 Department of Astronomy, University of Florida, Gainesville, FL 32611, USA \\ 6 Department of Physics and Astronomy, University College London, Gower Street, London WC1E 6BT, UK \\ 7 Department of Physics and Astronomy, University of California, Los Angeles, CA 90095-1547, USA \\ 8 Steward Observatory, University of Arizona, 933 North Cherry Avenue, Tucson, AZ 85721, USA \\ 9 Infrared Processing and Analysis Center, California Institute of Technology, MC 220-6, Pasadena, CA 91125, USA \\ ${ }^{10}$ Harvard-Smithsonian Center for Astrophysics, 60 Garden Street, Cambridge, MA 02138, USA \\ 11 Max-Planck-Institut für Radioastronomie, Auf dem Hügel 69, 53121 Bonn, Germany \\ 12 Department of Astronomy and Department of Physics, University of Illinois, 1002 West Green Street, Urbana, IL 61801, USA \\ 13 Department of Physiscs, Oxford University, Denis Wilkinson Building, Keble Road, Oxford, OX1 3RH, UK
}

Received 13 November 2015 / Accepted 6 January 2016

\section{ABSTRACT}

\begin{abstract}
We present ALMA detections of the [NII] $205 \mu \mathrm{m}$ and $\mathrm{CO}(12-11)$ emission lines, and the tentative detection of $[\mathrm{CI}]{ }^{3} \mathrm{P}_{1}-{ }^{3} \mathrm{P}_{0}$ for the strongly lensed $(\mu=5.7 \pm 0.5)$ dusty, star-forming galaxy SPT-S J213242-5802.9 (hereafter SPT2132-58) at $z=4.77$. The [NII] and $\mathrm{CO}(12-11)$ lines are detected at 11.5 and $8.5 \sigma$ levels, respectively, by our band 6 observations. The [CI] line is detected at $3.2 \sigma$ after a reanalysis of existing band 3 data. The $[\mathrm{CI}]$ luminosity implies a gas mass of $(3.8 \pm 1.2) \times 10^{10} M_{\odot}$, and, consequently, a very short depletion timescale of $34 \pm 13 \mathrm{Myr}$ and a CO luminosity to gas mass conversion factor $\alpha_{\mathrm{CO}}$ of $1.0 \pm 0.3 M_{\odot}\left(\mathrm{K} \mathrm{km} \mathrm{s}^{-1} \mathrm{pc}^{2}\right)^{-1}$. SPT2132-58 is an extreme starburst with an intrinsic star formation rate of $1100 \pm 200 M_{\odot} / \mathrm{yr}$. We find a [CII]/[NII] ratio of $26 \pm 6$, which is the highest ratio reported at $z>4$. This suggests that SPT2132-58 hosts an evolved interstellar medium $\left(0.5 Z_{\odot}<Z<1.5 Z_{\odot}\right)$, which may be dominated by photodissociation regions. The $\mathrm{CO}(2-1)$ and $\mathrm{CO}(5-4)$ transitions have lower $\mathrm{CO}$ to far-infrared ratios than local and high-redshift samples, while $\mathrm{CO}(12-11)$ is similar to these samples, suggesting the presence of an additional very excited component or an active galactic nucleus.
\end{abstract}

Key words. galaxies: starburst - galaxies: ISM - galaxies: high-redshift - galaxies: star formation - submillimeter: galaxies

\section{Introduction}

The presence of a large population of massive, dusty galaxies hosting high star formation rates $\left(S F R>500 M_{\odot} / \mathrm{yr}\right)$ at high redshift (e.g. Smail et al. 1997; Blain et al. 2002; Chapman et al. 2005) cannot be explained by the standard semi-analytical models of galaxy formation and hydrodynamical simulations (e.g. Cousin et al. 2015; Sparre et al. 2015). Recently, several of these extreme star-forming galaxies have been found at $z>4$ (e.g. Riechers et al. 2013; Vieira et al. 2013; Dowell et al. 2014; Smolčić et al. 2015), indicating extremely quick assembly of these structures. Their high SFRs suggest the presence of large gas reservoirs and their large dust content indicates that their interstellar medium (ISM) is metal enriched. This raises the question of how such a massive and mature ISM can be built so early?

Studying the ISM of these $z>4$ galaxies is difficult using the rest-frame optical because of the strong dust attenuation (da Cunha et al. 2015). The dust continuum and the (sub-)millimeter lines from the ISM are thus the main information we can obtain and the continuum and [CII] can now be routinely detected by ALMA (e.g., Scoville et al. 2014; Capak et al. 2015). The [CII]/[NII] line ratio was proposed as a new diagnostic of the ISM in star-forming galaxies (Nagao et al. 2012), but its use is hampered by the faintness of the [NII] line requiring long integration times, even with ALMA (Nagao et al. 2012; Decarli et al. 2014). This problem can be solved by targeting sources amplified by gravitational lensing. The sample of strongly lensed sources found by the South Pole Telescope (SPT; Carlstrom et al. 2011) is particularly suited for these follow-up studies because of its high median redshift $(\langle z\rangle=3.9$; Strandet et al. 2016).

In this Letter, we report the detections of the [NII], $\mathrm{CO}(12-11)$, and $[\mathrm{CI}]{ }^{3} \mathrm{P}_{1}-{ }^{3} \mathrm{P}_{0}$ lines in SPT-S J213242-5802.9 at $z=4.768$ (hereafter, SPT2132-58; Weiß et al. 2013; Gullberg et al. 2015). We assume the Planck Collaboration XIII (2015) cosmology $\left(H_{0}=67.8 \mathrm{~km} \mathrm{~s}^{-1} \mathrm{Mpc}^{-1}, \Omega_{\mathrm{m}}=0.308\right)$.

\section{Data}

SPT2132-58 was observed with ALMA in band 6 on February 232014 (2012.1.00994.S, PI: De Breuck) with a 

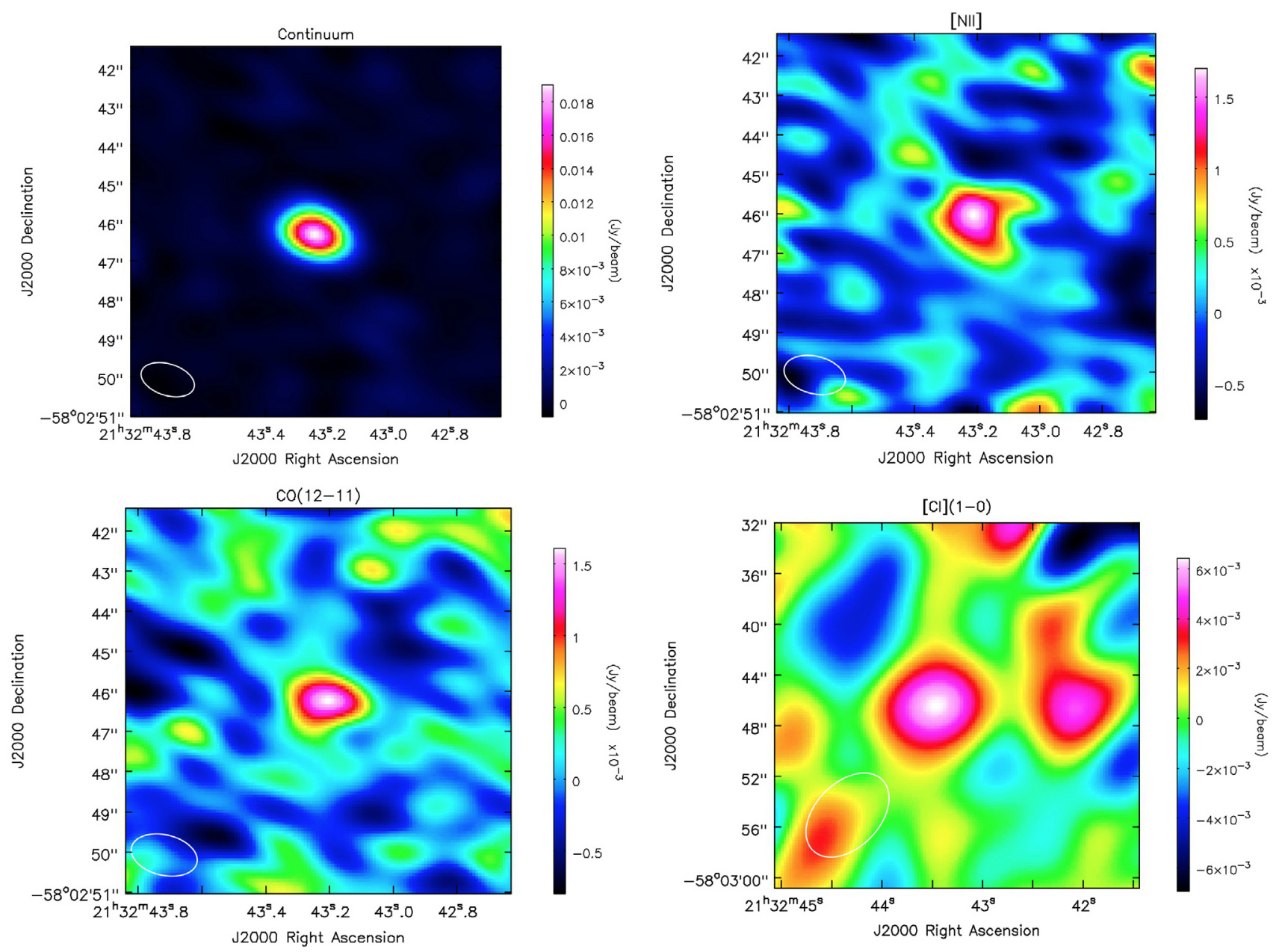

Fig. 1. Maps of the continuum (top left), the [NII] line (top right), the $\mathrm{CO}(12-11)$ line (bottom left), and the [CI](1-0) line (bottom right). The size of the beam is indicated in the lower left corner. [NII] and $\mathrm{CO}(12-11)$ were integrated between -300 and $+300 \mathrm{~km} \mathrm{~s}^{-1}$. Since the $[\mathrm{CI}](1-0)$ line is narrower and fainter, it was integrated between -100 and $+100 \mathrm{~km} \mathrm{~s}^{-1}$ to maximize the signal-to-noise ratio. The beam in band 3 ([CI](1-0)) is much larger than in band 6 . We thus stretch the $x$ - and $y$-axis by a factor 3 .

relatively compact configuration (minimal baseline $=15 \mathrm{~m}$, $\max =399 \mathrm{~m}, \mathrm{rms}=140 \mathrm{~m})$ and good weather $(\mathrm{PWV}=0.9 \mathrm{~mm})$. The on-source integration time is $470 \mathrm{~s}$. The data were calibrated by the standard ALMA pipeline. The four spectral windows are centred on 237.6, 239.5, 252.9, and 254.8 GHz. Each spectral window has 480 channels and covers $1875 \mathrm{MHz}$. We produced the images of our source with CASA (McMullin et al. 2007) using the clean routine, and we chose a natural weighting to optimize the sensitivity (see Sect.3). This results in a synthesized beam size of $1.55^{\prime \prime} \times 0.91^{\prime \prime}$. The typical RMS noise is $0.36 \mathrm{mJy} /$ beam for the continuum (combining the four spectral windows $)$ and $1.6 \mathrm{mJy} / \mathrm{beam} /\left(25 \mathrm{~km} \mathrm{~s}^{-1}\right.$ channel) for the spectra. The spectra were extracted using an elongated aperture twice as large in diameter than the beam to avoid losing flux because our source is marginally resolved. We also produced a continuum image with a Briggs weighting with robust $=0.5$, since the signal-to-noise ratio is already very high $(S N R=52$; see Fig. 1$)$.

In addition, we reanalyzed band 3 data obtained during the cycle 0 (Weiß et al. 2013) using the same approach (see Fig. 1). We used a natural weighting instead of a Briggs weighting with robust $=0.5$. The beam size is $7.7^{\prime \prime} \times 4.9^{\prime \prime}$ and the noise is $3.2 \mathrm{mJy} /$ beam $/\left(50 \mathrm{~km} \mathrm{~s}^{-1}\right.$ channel $)$. The natural weighting reduces the noise in the spectrum and improves the signal-to-noise ratio of the $[\mathrm{CI}]$ line from 2.9 to 3.2 .

\section{Results}

The $[\mathrm{NII}]$ and $\mathrm{CO}(12-11)$ lines are detected unambiguously at 11.5 and $8.5 \sigma$, respectively (see Fig. 2). The reanalysis of the cycle 0 data allowed us to obtain a tentative detection of [CI] at $3.2 \sigma$. The fluxes of the lines are listed in Table 1. Finally, we measured the flux density of the source and found an integrated flux $26.4 \pm 0.5 \mathrm{mJy}$ at $246 \mathrm{GHz}$. The [NII] and $\mathrm{CO}(12-11)$ lines were not excluded from this measurement, since ancillary bolometric data also include lines and their contribution is only $1.3 \%$ of the total continuum flux integrated over the four spectral windows. The source is marginally resolved along its major axis. The AIPS and CASA softwares provide a similar best estimate of the source size of $(0.6 \pm 0.2)^{\prime \prime}$. This value is consistent with the size in the image plane predicted by our best lens model (0.67"; Spilker et al., in prep.).

We compared the profiles of these new detections with the previously reported detections of $\mathrm{CO}(5-4)$ by Weiß et al. (2013), [CII] by Gullberg et al. (2015), and $\mathrm{CO}(2-1)$ by Aravena et al. (2016). All the lines are compatible with a single Gaussian function (highest reduced $\chi^{2}$ is 1.4 , which is compatible at $1 \sigma$ with unity considering their number of degrees of freedom). The results of the fits are given in Table 1. The [NII], [CII], $\mathrm{CO}(5-4)$, and $\mathrm{CO}(2-1)$ lines have similar full widths at half maximum 
Table 1. Summary of the properties of the detected lines of SPT2132-58.

\begin{tabular}{lllllcll}
\hline \hline Line & $\begin{array}{l}v_{\text {rest }} \\
\mathrm{GHz}\end{array}$ & $\begin{array}{l}I_{\text {line }}{ }^{a} \\
\mathrm{Jy} \mathrm{km} \mathrm{s}^{-1}\end{array}$ & $\begin{array}{l}\mu L_{\text {line }}^{\prime} \\
10^{10} \mathrm{~K} \mathrm{~km}^{-1} \mathrm{pc}^{2}\end{array}$ & $\begin{array}{l}\mu L_{\text {line }} \\
10^{8} L_{\odot}\end{array}$ & $\begin{array}{c}F W H M \\
\mathrm{~km} \mathrm{~s}^{-1}\end{array}$ & Instrument & Reference \\
\hline $\mathrm{CO}(2-1)$ & 230.54 & $0.85 \pm 0.07$ & $18.38 \pm 1.51$ & $0.71 \pm 0.06$ & $225 \pm 17$ & ATCA & Aravena et al. (2016) \\
{$[\mathrm{CI}(1-0)]$} & 492.16 & $0.86 \pm 0.27$ & $4.07 \pm 1.29$ & $1.54 \pm 0.49$ & $\ldots$ & ALMA band 3 & This work \\
$\mathrm{CO}(5-4)$ & 576.35 & $1.41 \pm 0.26$ & $4.88 \pm 0.90$ & $2.96 \pm 0.54$ & $231 \pm 70$ & ALMA band 3 & Weiß et al. (2013) \\
$\mathrm{CO}(12-11)$ & 1383.24 & $1.36 \pm 0.16$ & $0.82 \pm 0.10$ & $6.84 \pm 0.81$ & $331 \pm 78$ & ALMA band 6 & This work \\
{$[\mathrm{NII}]$} & 1461.10 & $1.73 \pm 0.15$ & $0.93 \pm 0.08$ & $9.20 \pm 0.79$ & $240 \pm 41$ & ALMA band 6 & This work \\
{$[\mathrm{CII}]$} & 1900.54 & $34.90 \pm 6.90$ & $11.10 \pm 2.19$ & $241.53 \pm 47.75$ & $212 \pm 43$ & APEX/FLASH & Gullberg et al. (2015) \\
\hline
\end{tabular}

Notes. These apparent luminosities have not been corrected for lensing magnification. ${ }^{(a)}$ The integrated line flux $I_{\mathrm{CO}}$ is computed between -1.5 and 1.5 FWHM.

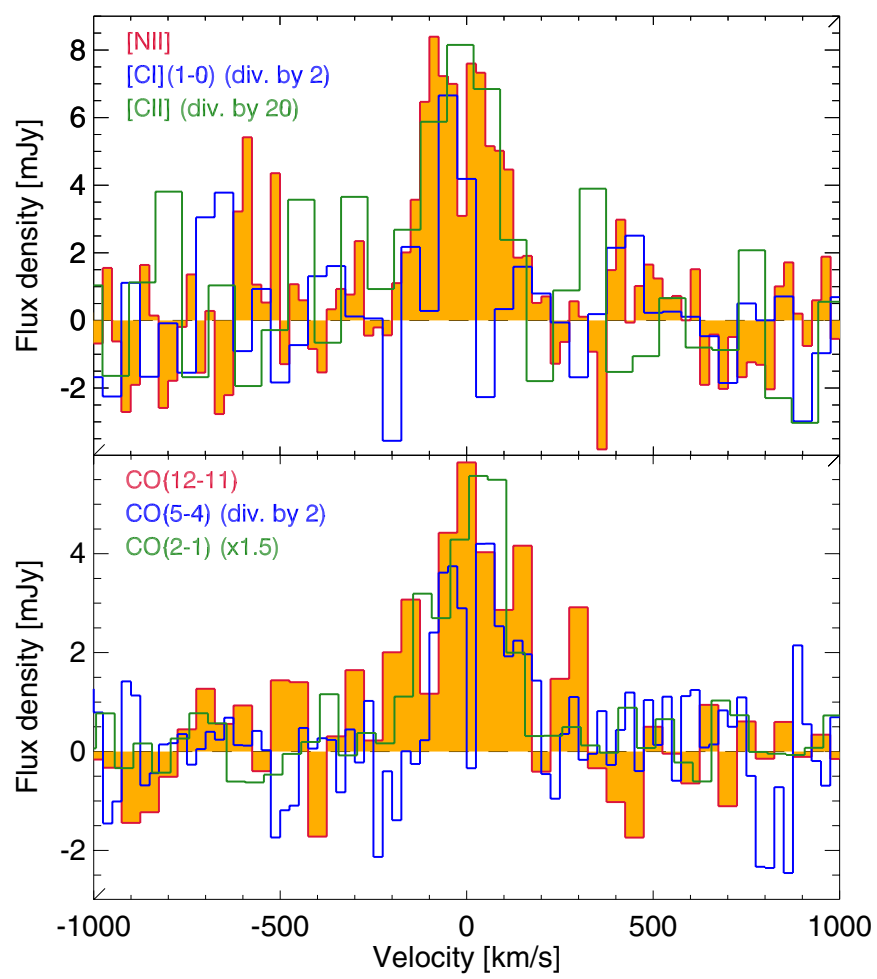

Fig. 2. Upper panel: profile of the fine structure lines of SPT2132-58 ([NII] in orange, $[\mathrm{CI}](1-0)$ in blue, and $[\mathrm{CII}]$ in green). Lower panel: profile of the CO lines (12-11 in orange, 5-4 in blue, and 2-1 in green).

(FWHMs). The $\mathrm{CO}(12-11)$ line is slightly wider than the other lines $(1.0 \sigma$ compared to $[\mathrm{NII}])$. The low SNR of $[\mathrm{CI}]$ prohibits us from determining the FWHM.

\section{Discussion}

\subsection{Star formation efficiency and nature of the source}

SPT2132-58 is gravitationally magnified by another galaxy on the line of sight. Our best model, based on the ALMA cycle-0, high-resolution follow-up of the source, estimates that the magnification is $\mu=5.7 \pm 0.5$ (Spilker et al., in prep.). We determined its apparent total infrared luminosity $\left(\mu L_{\mathrm{IR}(8-1000 \mu \mathrm{m})}=(6.4 \pm\right.$ $\left.1.0) \times 10^{13} L_{\odot}\right)$ and the far-infrared luminosity $\left(\mu L_{\mathrm{FIR}(50-300 \mu \mathrm{m})}=\right.$ $\left.(3.9 \pm 0.6) \times 10^{13} L_{\odot}\right)$ by fitting the continuum measurements made by ALMA band 3, SPT, LABOCA, Herschel/SPIRE (Strandet et al., in prep.), and ALMA band 6 (Sect. 3 and Fig. 3). We used the starburst templates of Magdis et al. (2012) to fit the continuum. The quality of the fit is $\operatorname{good}\left(\chi_{\mathrm{red}}^{2}=1.1\right)$, but the SPT point at $220 \mathrm{GHz}$ exhibits a $2 \sigma$ excess. This tension

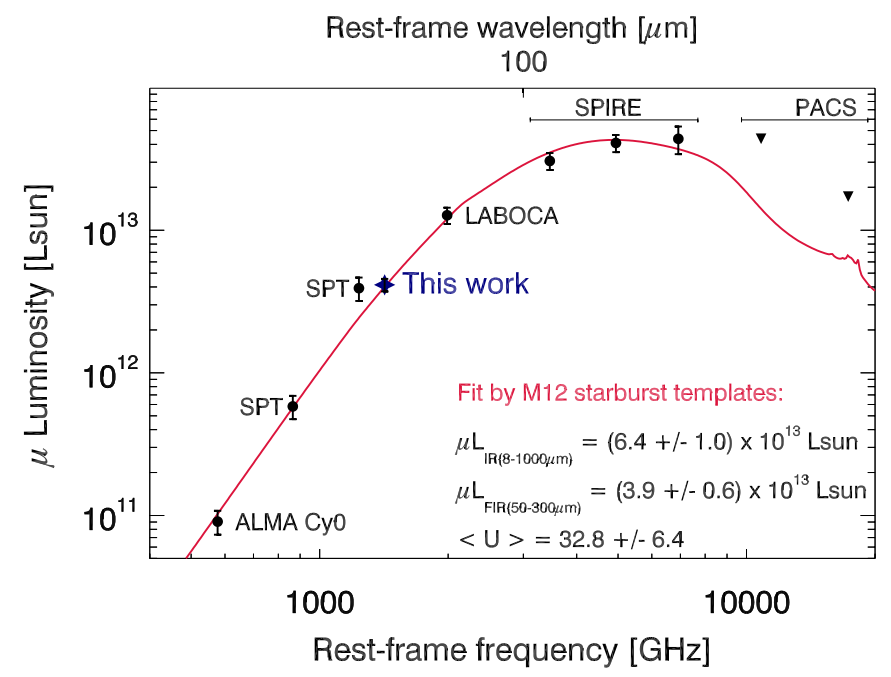

Fig. 3. SED of SPT2132-58, including a compilation of measurements from Strandet et al. (in prep.), (filled black circles for detections, downwards triangles for $3 \sigma$ upper limits) and our new ALMA Cycle 1 continuum measurement (blue filled star), and best fit by the starburst templates of the Magdis et al. (2012) model (red line). The $\langle\mathrm{U}\rangle$ parameter is the mean intensity of the radiation field heating the dust compared to the solar neighbourhood.

could be explained by a peak of noise at the position of our source and not by an astrophysical contaminant since no excess is found in the other SPT band. We found an apparent luminosity $\mu L_{\mathrm{IR}}$ of $(6.4 \pm 1.0) \times 10^{13} L_{\odot}$. Because of the high dust temperature of the source $\left(T_{\text {dust }}=41 \mathrm{~K}\right.$, Aravena et al. 2016), the CMB has a negligible impact on this measurement (da Cunha et al. 2013). The intrinsic $L_{\mathrm{IR}}$ is thus around $10^{13} L_{\odot}$ putting this source at the edge of the hyper-luminous infrared galaxy (HyLIRG; $L_{\mathrm{IR}}>10^{13} L_{\odot}$ ) category. This corresponds to an intrinsic SFR of $1120 \pm 200 M_{\odot} / \mathrm{yr}$ (Kennicutt 1998 assuming a Chabrier 2003 initial mass function). This SFR range is expected to be populated only by episodic starbursts, according to the Béthermin et al. (2012) model.

We estimated the gas mass from the $[\mathrm{CI}]$ emission (Papadopoulos \& Greve 2004). Assuming the abundance and excitation factors of Alaghband-Zadeh et al. (2013), we found an apparent gas mass $\mu M_{\text {gas }}$ of $(21.5 \pm 6.7) \times 10^{10} M_{\odot}$ and a demagnified gas mass of $(3.8 \pm 1.2) \times 10^{10} M_{\odot}$. A systematic analysis of all [CI] lines detected in SPT sources and a detailed discussion about the accuracy of [CI]-derived gas mass will be presented in Bothwell et al. (in prep.). We can also estimate the $\alpha_{\mathrm{CO}}$ of this object from the $L_{\mathrm{CO}(2-1)}^{\prime}$ line luminosity measured by Aravena et al. (2016), converting it into $L_{\mathrm{CO}(1-0)}^{\prime}$ using the mean SMG 


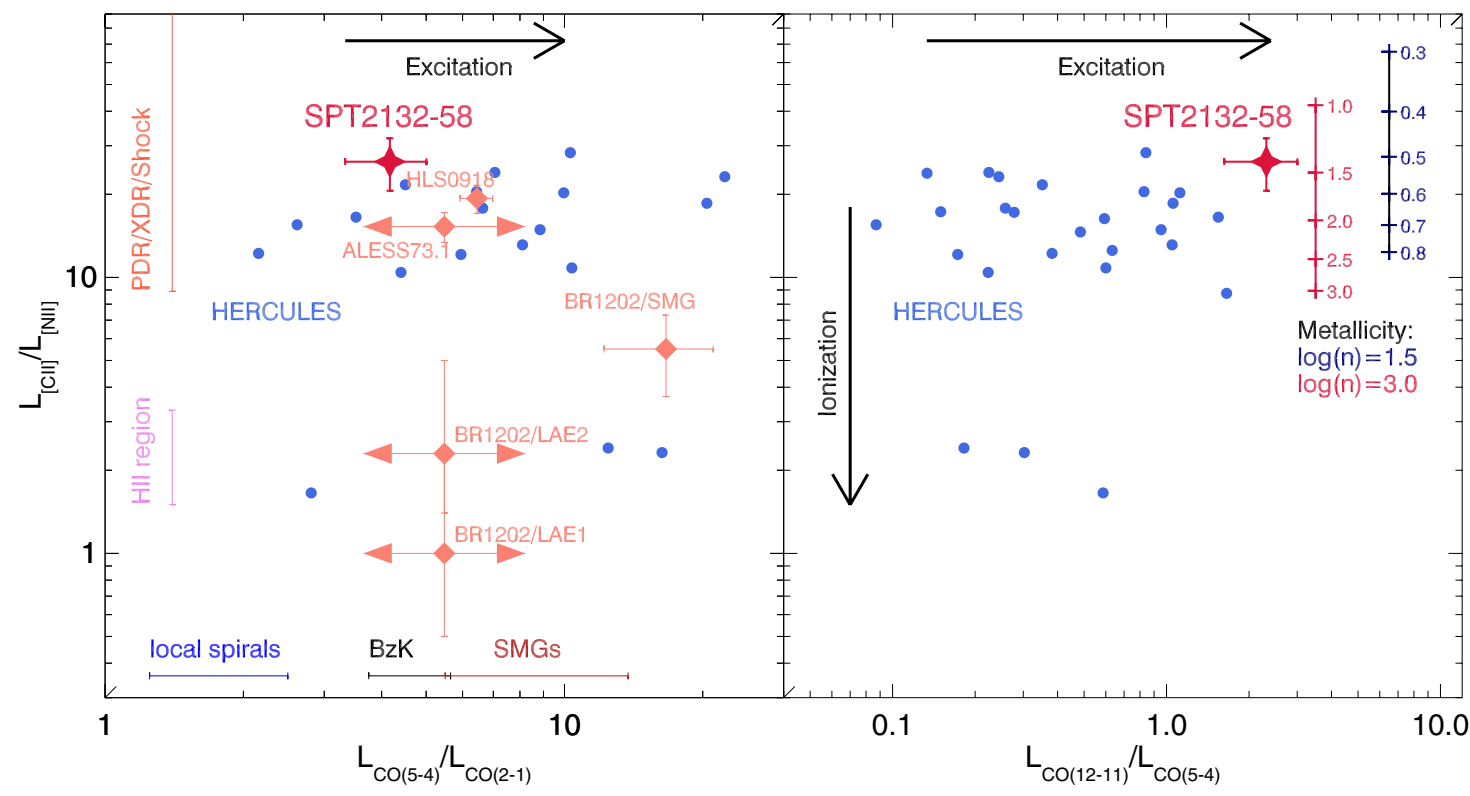

Fig. 4. $\mathrm{CO}$ excitation $(\mathrm{CO}(5-4) / \mathrm{CO}(2-1)$ in the left panel and $\mathrm{CO}(12-11) / \mathrm{CO}(5-4)$ in the right panel) versus $[\mathrm{CII}] /[\mathrm{NII}]$ luminosity ratio (the ratio reported for ALESS73.1 is computed using the updated [CII] flux of De Breuck et al. 2014). Red star: SPT2132-58. Salmon diamonds: other high- $z$ sources from the literature (see text). Blue circles: HERCULES local sample (Rosenberg et al. 2015). [NII] luminosities are from Kamenetzky et al. (2015). The left and right arrows indicate that this source does not have available measurement of its CO ratio. The range of $\mathrm{CO}(5-4) / \mathrm{CO}(2-1)$ ratio for various samples is indicated in the bottom of the left panel (Daddi et al. 2015). The ranges of ratio expected for HII and PDR/XDR/shock dominated regions from Decarli et al. (2014) are indicated on the left. The metallicity estimated from [CII]/[NII], following the Nagao et al. (2012) method, is indicated on the right for $\log (n)=1.5$ (navy) and $\log (n)=3.0$ (blue). We assume $\log \left(U_{\mathrm{HII}}\right)=-3.5$ for both.

ratio (Carilli \& Walter 2013), and assuming the gas mass provided by $[\mathrm{CI}]$. We find $\alpha_{\mathrm{CO}}=0.99 \pm 0.32 M_{\odot}\left(\mathrm{K} \mathrm{km} \mathrm{s}^{-1} \mathrm{pc}^{2}\right)^{-1}$. This agrees with $\alpha_{\mathrm{CO}}$ of $0.9 \pm 0.3\left(\mathrm{~K} \mathrm{~km} \mathrm{~s}^{-1} \mathrm{pc}^{2}\right)^{-1}$ derived by Aravena et al. (2016) from the dust mass assuming a gas-to-dust mass ratio of 100 . This value is compatible with the classical $\sim 0.8 M_{\odot}\left(\mathrm{K} \mathrm{km} \mathrm{s}^{-1} \mathrm{pc}^{2}\right)^{-1}$ found for local compact starbursts (Downes \& Solomon 1998).

The depletion timescale associated with this gas mass and SFR is $34 \pm 13$ Myr. This is a factor of 3 shorter than typical strong starbursts and more than an order of magnitude less than normal star-forming galaxies at this redshift (e.g. Béthermin et al. 2015). The short depletion timescale indicates that this source is an extreme starburst with a moderate gas content, but a very high star formation efficiency. The depletion timescale could however be slightly underestimated if this source is observed at the end of the starburst. In this case, the SFR would be quickly decreasing. The SFR derived from $L_{\mathrm{IR}}$ would thus be lower than the actual SFR, since this tracer is sensitive to the SFR in the last 10-100 Myr (Kennicutt 1998).

\subsection{Properties of ISM}

SPT2132-58 is one of the few high-redshift sources that have been detected in both [CII] and [NII] $205 \mu \mathrm{m}(y$ axis of Fig. 4). The previous detections were obtained in HLS J091828.6+514223 (Combes et al. 2012; Rawle et al. 2014), ALESS73.1 (Nagao et al. 2012), and one SMG and two LAEs (tentative detections) in the BR1202-0725 system (Decarli et al. 2014). We find a [CII]/[NII] luminosity ratio of $26.3 \pm 5.7$ in SPT2132-58, which is the highest reported so far at high redshift. HLS J0918 has a similar ratio within $1 \sigma$, but ALESS73.1 is two times lower. The members of the BR1202-0725 system have a much lower ratio (4-26 times). Finally, we compared SPT213258 with the HERCULES sample of local (U)LIRGs (Rosenberg et al. 2015). All but three of the 29 HERCULES sources have a lower ratio. However, the $[\mathrm{CII}] /[\mathrm{NII}]$ ratio of SPT2132-58 is not uncommon in the local Universe and is close to, e.g. the mean value of 30 found by Spinoglio et al. (2015) in local Seyfert galaxies.

The $[\mathrm{CII}] /[\mathrm{NII}]$ ratio of SPT2132-58 corresponds to a PDR/XDR/shock-dominated regime according to Decarli et al. (2014). For galaxies in this regime, the [CII] originates from both HII regions and external layers of PDRs, while [NII] only comes from HII regions. Using a model that takes both PDR and HII regions into account, Nagao et al. (2012) showed that the observed $[\mathrm{CII}] /[\mathrm{NII}]$ ratio depends on metallicity. This occurs since the PDR/HII fraction of [CII] varies with metallicity. Other parameters affecting this ratio include density and, to a lesser extent, the radiation field. Figure 4 shows the predictions of Nagao et al. (2012) for both a low (navy) and a high (red) density, but only for a single ionization parameter $\log \left(U_{\mathrm{HII}}\right)=-3.5$. The $[\mathrm{CII}] /[\mathrm{NII}]$ ratio of SPT2132-58 corresponds to a metallicity in the 0.5 to $1.5 Z_{\odot}$ range. While we could have expected a very low metallicity at such a high redshift, our source is not far from solar metallicity. Nevertheless, our source has a slightly higher $[\mathrm{CII}] /[\mathrm{NII}]$ ratio than the HERCULES sample. Although the $[\mathrm{CII}] /[\mathrm{NII}]$ ratio depends on several other physical parameters (e.g. density), the higher ratio found in SPT2132-58 suggests a slightly lower metallicity than in local starbursts.

We also compared the CO excitation with the samples cited previously ( $x$-axis of Fig.4). SPT2132-58 has a $\mathrm{CO}(5-4) / \mathrm{CO}(2-1)$ ratio close to the average of the HERCULES sample. Assuming $T_{\text {kin }}=40 \mathrm{~K}$, the CMB at $z=4.77$ has only an impact of $15 \%$ on this ratio (da Cunha et al. 2013). The $\mathrm{CO}(5-4) / \mathrm{CO}(2-1)$ ratio lies at the border between the value found for BzK galaxies and SMGs (Daddi et al. 2015) and is thus standard for a high-redshift galaxy. However, these two lines have a lower $L_{\mathrm{CO}} / L_{\mathrm{FIR}}(50-300 \mu \mathrm{m})$ ratio than the other samples of DSFGs by a factor of 2 and 3 for the $\mathrm{CO}(2-1)$ and $\mathrm{CO}(5-4)$ transitions, respectively (see Fig. 5). This is compatible with the 


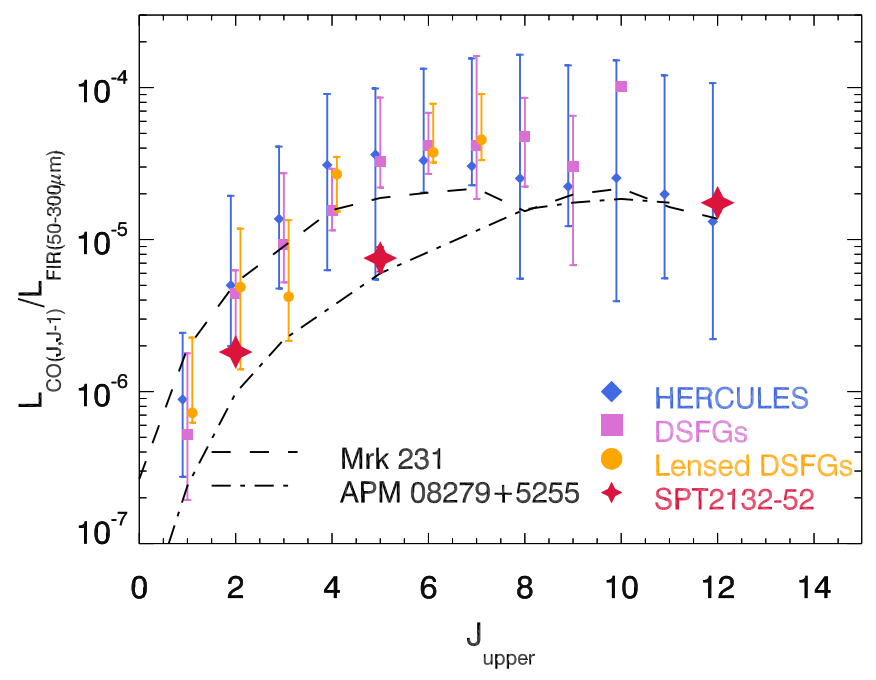

Fig. 5. CO spectral line energy distribution (SLED) of SPT2132-58 normalized by $L_{\mathrm{FIR}}$. Our results are compared with three data compilations of Greve et al. (2014; the error bars indicate the lowest and highest value of their samples). The dashed line is the SLED of Mrk231 (Rosenberg et al. 2015) and the dash-dotted line is the SLED of APM $08279+5255$ (Downes et al. 1999; Weiß et al. 2007; Riechers et al. 2009; Bradford et al. 2011).

moderate gas content and high SFR found in Sect. 4.1, since $L_{\mathrm{CO}}$ correlates with the gas content and SFR with $L_{\mathrm{FIR}(50-300 \mu \mathrm{m})}$. The $\mathrm{CO}(5-4)$ line is also a factor of $3.2 \pm 0.4$ lower than the average $\mathrm{CO}(5-4)$ flux of the SPT sample measured by stacking in Spilker et al. (2014).

In contrast, the $L_{\mathrm{CO}(12-11)}^{\prime} / L_{\mathrm{FIR}(50-300 \mu \mathrm{m})}$ ratio is similar to the HERCULES sample, but the $\mathrm{CO}(12-11) / \mathrm{CO}(5-4)$ ratio of SPT2132-58 is higher than all of the objects of the HERCULES sample. There is thus a deficit of flux of the lowand mid- $J$ CO lines, but a strong $\mathrm{CO}(12-11)$ line that may be emitted by different regions and/or excited by another mechanism. This could involve an active galactic nucleus (AGN, e.g. Gallerani et al. 2014) and/or regions with a particularly high radiation field. This effect could possibly be amplified by differential magnification (Serjeant 2012). If a very excited compact region is located on a caustic and has its flux magnified by a much larger factor than the rest of the system, the apparent ratio between a high- $J$ and a low- $J$ CO line can be higher than the intrinsic ratio. In Fig. 5, we compare the SLED of SPT2132-58 with the AGN-host galaxies Mrk231 and APM 08279+5255. The SPT2132-58 has a similar $L_{\mathrm{CO}(12-11)} / L_{\mathrm{FIR}}(50-300 \mu \mathrm{m})$ ratio to these two objects. At $J \leq 5$, there is a factor of 5 difference between Mrk231, which matches the properties of the HERCULES sample, and APM 08279+5255, which is a strong outlier. SPT2132-58 has intermediate properties and its SLED does not allow us to reach a conclusion about the presence or not of an AGN. Future [OI] or mid-infrared observations are required to test the hypothesis.

\section{Conclusion}

We investigated the physical properties of SPT2132-58, a lensed source at $z=4.77$. We determined that this source is an HyLIRG $\left(S F R=1120 \pm 200 M_{\odot} / \mathrm{yr}\right)$ with a relatively modest gas reservoir $\left(3.8 \pm 1.2 \times 10^{10} M_{\odot}\right)$ and a very short gas depletion timescale ( $34 \pm 13 \mathrm{Myr})$, indicating a very intense starburst. The $\alpha_{\mathrm{CO}}$ conversion factor $(0.99 \pm 0.32)$ is similar with that measured in starbursts at lower redshift. The $[\mathrm{CII}] /[\mathrm{NII}]$ ratio $(26.3 \pm 5.7)$ is the highest found in a high-redshift galaxy and indicates a chemically-evolved ISM $\left(0.5 Z_{\odot}<Z<1.5 Z_{\odot}\right)$. This source is an important laboratory to understand the extremely quick stellar mass assembly of the first massive galaxies.

Acknowledgements. We thanks Roberto Decarli for his very useful suggestions, especially about the choices of axes for Fig. 4, and the anonymous referee for her/his very constructive comments. This paper makes use of the following ALMA data: ADS/JAO.ALMA\#2011.0.00957.S and ADS/JAO.ALMA\#2012.1.00994.S. ALMA is a partnership of ESO (representing its member states), NSF (USA) and NINS (Japan), together with NRC (Canada), NSC and ASIAA (Taiwan), and KASI (Republic of Korea), in cooperation with the Republic of Chile. The Joint ALMA Observatory is operated by ESO, AUI/NRAO, and NAOJ. The National Radio Astronomy Observatory is a facility of the National Science Foundation operated under cooperative agreement by Associated Universities, Inc. The SPT is supported by the National Science Foundation through grant PLR- 1248097, with partial support through PHY-1125897, the Kavli Foundation and the Gordon and Betty Moore Foundation grant GBMF 947. J.D.V., K.C.L., D.P.M., and J.S.S. acknowledge support from the US National Science Foundation under grant No. AST-1312950.

\section{References}

Alaghband-Zadeh, S., Chapman, S. C., Swinbank, A. M., et al. 2013, MNRAS, 435,1493

Aravena, M., Spilker, J. S., \& et al. 2016, ApJ, submitted

Béthermin, M., Daddi, E., Magdis, G., et al. 2012, ApJ, 757, L23

Béthermin, M., Daddi, E., Magdis, G., et al. 2015, A\&A, 573, A113

Blain, A. W., Smail, I., Ivison, R. J., Kneib, J.-P., \& Frayer, D. T. 2002, Phys. Rep., 369, 111

Bradford, C. M., Bolatto, A. D., Maloney, P. R., et al. 2011, ApJ, 741, L37

Capak, P. L., Carilli, C., Jones, G., et al. 2015, Nature, 522, 455

Carilli, C. L., \& Walter, F. 2013, ARA\&A, 51, 105

Carlstrom, J. E., Ade, P. A. R., Aird, K. A., et al. 2011, PASP, 123, 568

Chabrier, G. 2003, PASP, 115, 763

Chapman, S. C., Blain, A. W., Smail, I., \& Ivison, R. J. 2005, ApJ, 622, 772

Combes, F., Rex, M., Rawle, T. D., et al. 2012, A\&A, 538, L4

Cousin, M., Lagache, G., Bethermin, M., Blaizot, J., \& Guiderdoni, B. 2015, A\&A, 575, A32

da Cunha, E., Groves, B., Walter, F., et al. 2013, ApJ, 766, 13

da Cunha, E., Walter, F., Smail, I. R., et al. 2015, ApJ, 806, 110

Daddi, E., Dannerbauer, H., Liu, D., et al. 2015, A\&A, 577, A46

De Breuck, C., Williams, R. J., Swinbank, M., et al. 2014, A\&A, 565, A59

Decarli, R., Walter, F., Carilli, C., et al. 2014, ApJ, 782, L17

Dowell, C. D., Conley, A., Glenn, J., et al. 2014, ApJ, 780, 75

Downes, D., \& Solomon, P. M. 1998, ApJ, 507, 615

Downes, D., Neri, R., Wiklind, T., Wilner, D. J., \& Shaver, P. A. 1999, ApJ, 513, L1

Gallerani, S., Ferrara, A., Neri, R., \& Maiolino, R. 2014, MNRAS, 445, 2848

Greve, T. R., Leonidaki, I., Xilouris, E. M., et al. 2014, ApJ, 794, 142

Gullberg, B., De Breuck, C., Vieira, J. D., et al. 2015, MNRAS, 449, 2883

Kamenetzky, J., Rangwala, N., Glenn, J., Maloney, P. R., \& Conley, A. 2015, ApJ, submitted [arXiv: 1508.05102]

Kennicutt, Jr., R. C. 1998, ApJ, 498, 541

Magdis, G. E., Daddi, E., Béthermin, M., et al. 2012, ApJ, 760, 6

McMullin, J. P., Waters, B., Schiebel, D., Young, W., \& Golap, K. 2007, ASP Conf. Ser., 376, 127

Nagao, T., Maiolino, R., De Breuck, C., et al. 2012, A\&A, 542, L34

Papadopoulos, P. P., \& Greve, T. R. 2004, ApJ, 615, L29

Planck Collaboration XIII. 2015, A\&A, submitted [arXiv : 1502.01589]

Rawle, T. D., Egami, E., Bussmann, R. S., et al. 2014, ApJ, 783, 59

Riechers, D. A., Walter, F., Carilli, C. L., \& Lewis, G. F. 2009, ApJ, 690, 463

Riechers, D. A., Bradford, C. M., Clements, D. L., et al. 2013, Nature, 496, 329

Rosenberg, M. J. F., van der Werf, P. P., Aalto, S., et al. 2015, ApJ, 801, 72

Scoville, N., Aussel, H., Sheth, K., et al. 2014, ApJ, 783, 84

Serjeant, S. 2012, MNRAS, 424, 2429

Smail, I., Ivison, R. J., \& Blain, A. W. 1997, ApJ, 490, L5

Smolčić, V., Karim, A., Miettinen, O., et al. 2015, A\&A, 576, A127

Sparre, M., Hayward, C. C., Springel, V., et al. 2015, MNRAS, 447, 3548

Spilker, J. S., Marrone, D. P., Aguirre, J. E., et al. 2014, ApJ, 785, 149

Spinoglio, L., Pereira-Santaella, M., Dasyra, K. M., et al. 2015, ApJ, 799, 21

Strandet, M., de Breuck, C., Vieira, J., Weiss, A., \& SPT collaboration 2016, ApJ, submitted

Vieira, J. D., Marrone, D. P., Chapman, S. C., et al. 2013, Nature, 495, 344

Weiß, A., Downes, D., Neri, R., et al. 2007, A\&A, 467, 955

Weiß, A., De Breuck, C., Marrone, D. P., et al. 2013, ApJ, 767, 88 